\title{
Regularities with random interactions in energy centroids defined by group symmetries
}

\author{
V. K. B. Kota \\ Physical Research Laboratory, Ahmedabad 380 009, India
}

\begin{abstract}
Regular structures generated by random interactions in energy centroids defined over irreducible representations (irreps) of some of the group symmetries of the interacting boson models $s d \mathrm{IBM}, s d g \mathrm{IBM}$, $s d$ IBM- $T$ and $s d$ IBM-ST are studied by deriving trace propagations equations for the centroids. It is found that, with random interactions, the lowest and highest group irreps in general carry most of the probability for the corresponding centroids to be lowest in energy. This generalizes the result known earlier, via numerical diagonalization, for the more complicated fixed spin $(J)$ centroids where simple trace propagation is not possible.
\end{abstract}

Typeset using REVTEX 
Johnson, Bertsch and Dean in 1998 [1], using the nuclear shell model found that random two-body interactions lead to ground states, for even-even nuclei, having spin $0^{+}$with very high probability. Similarly, Bijker and Frank [2] using the interacting boson model with $s$ and $d$ bosons ( $s d \mathrm{IBM})$ showed that random interactions generate vibrational and rotational structures with high probability. Further studies using the shell model, fermions in one or two $j$ orbits, $s d, s p$ and $s d g$ IBM's, bosons in a single $\ell$ orbit etc., revealed statistical predominance of odd-even staggering in binding energies, $0^{+}, 2^{+}, 4^{+}, \ldots$ yrast sequence, regularities in ground states in parity distributions, occupation numbers and so on; see [3-10] and references therein. Notably, Zelevinsky et al [4] introduced the idea of geometric chaos as a basis for the regularities observed in shell model studies. Similarly, Zhao et al [8] developed a prescription based on sampling of the corners of the parameter space and Bijker and Frank [7,9] employed mean-field methods. The unexpected results for regularities with random interactions are reviewed in $[6,10]$. As Zhao et al stated [10]: "a more fundamental understanding of the robustness of $0_{g . s .}^{+}$dominance is still out of reach". Therefore, going beyond the ground states and near yrast levels, energy centroids, spectral widths and correlations among them are also being investigated by several groups $[4,5,11-13]$ as they are expected to give new insights into regularities generated by random interactions. For example, Zhao et al $[11,12]$ initiated the study of energy centroids and analyzed fixed- $L$ (fixed- $J$, JT) centroids in IBM's (in shell model) spaces. They found that $L_{\min }\left(\right.$ or $\left.J_{\min }\right)$ and $L_{\max }\left(\right.$ or $J_{\max }$ ) will be lowest with largest probabilities and others appear with negligible probability. Similarly Papenbrock and Weidenmüller [13] recently analyzed the structure of fixed- $J$ spectral widths for fermions in a single- $j$ shell.

An interesting and important question is the extension of the spin zero ground state dominance (and also other regular structures seen in shell model and IBM 
studies) to group theoretical models with hamiltonians preserving a symmetry higher than $J$ (or $L$ ). Similarly one may consider centroids and variances defined over good or broken symmetry subspaces. They open a new window to the regularities of many-body systems in the presence of random forces. Initiating work in this direction [9], recently random one plus two-body hamiltonians invariant with respect to $O\left(\mathcal{N}_{1}\right) \oplus O\left(\mathcal{N}_{2}\right)$ symmetry of a variety of interacting boson models are used to investigate the probability of occurrence of a given $\left(\omega_{1} \omega_{2}\right)$ irreducible representation (irrep) to be the ground state in even-even nuclei; $\left[\omega_{1}\right]$ and $\left[\omega_{2}\right]$ are symmetric irreps of $O\left(\mathcal{N}_{1}\right)$ and $O\left(\mathcal{N}_{2}\right)$ respectively. It is found that the $0^{+}$dominance observed in ground states of even-even nuclei extends to group irreps. The purpose of this paper and others to follow is to go beyond this and study regularities, generated by random interactions, in energy centroids, variances etc. defined over group irreps. Reported in this rapid communication are the results of a first analysis of energy centroids with examples from $s d$ IBM, $s d g$ IBM, $s d$ IBM- $T$ with the bosons carrying isospin $(T)$ and $s d$ IBM-ST with the bosons carrying spin-isospin $(S T)$ degrees of freedom. Before proceeding further, it is important to stress that energy centroids (also variances) can be calculated as a function of particle number $(m)$ and the quantum numbers labeling the group irreps, without recourse to the construction of the hamiltonian matrix. The principle used here is trace propagation, a subject introduced in the context of statistical nuclear spectroscopy by French $[14,15]$. Readers not interested in the details of group algebra and derivation of trace propagation equations for the energy centroids (given by Eqs. (5), (6), (7) and (8)), may leap ahead to the discussion of results starting just after Eq. (8).

Let us begin with the spectrum generating algebra (SGA), say $G_{1}$, of a group theoretical model with all the many particle states in the model belong to the irrep $\Gamma_{1}$ of $G_{1}$. For example, the SGA $G_{1}$ for $s d \mathrm{IBM}$ is $U(6)$. Now the average of an 
operator $\mathcal{O}(k)$ of maximum body rank $k$ over the irreps $\Gamma_{2}$ of a subalgebra $G_{2}$ of $G_{1}$ $\left(G_{2}\right.$ in general denotes a set of subalgebras contained in $G_{1}$ and $\Gamma_{2}$ denotes all their irreps) is defined by

$$
\langle\mathcal{O}(k)\rangle^{\Gamma_{1}, \Gamma_{2}}=\sum_{\beta} \sum_{\alpha \in \Gamma_{2}}\left\langle\Gamma_{1} \beta \Gamma_{2} \alpha|\mathcal{O}(k)| \Gamma_{1} \beta \Gamma_{2} \alpha\right\rangle /\left[\sum_{\beta} \sum_{\alpha \in \Gamma_{2}}\left\langle\Gamma_{1} \beta \Gamma_{2} \alpha|1| \Gamma_{1} \beta \Gamma_{2} \alpha\right\rangle\right]
$$

In Eq. (1), $\beta$ labels the multiple occurrence (multiplicity) of $\Gamma_{2}$ in a given $\Gamma_{1}$ irrep (i.e. in the reduction of $\Gamma_{1}$ to $\Gamma_{2}$ ). Removing the denominator in Eq. (1) gives the trace over $\left(\Gamma_{1}, \Gamma_{2}\right)$ space, i.e. $\operatorname{tr}[\mathcal{O}(k)]^{\Gamma_{1}, \Gamma_{2}}$. General theory for propagation of traces of operators over irreps of group symmetries is developed in Refs. [16-19]. In particular, Quesne [16] showed that, for $G_{1} \supset G_{2}$, trace propagation over the irreps $\Gamma_{1}$ and $\Gamma_{2}$ of $G_{1}$ and $G_{2}$ algebras is related to the so-called integrity basis of $G_{2}$ in $G_{1}$ which gives the minimal set of $G_{2}$ scalars in $G_{1}$. As discussed in Refs. [17,18], it is seen that in general the multiplicity of $\Gamma_{2}$ in a given $\Gamma_{1}$ irrep results in the propagation of matrix of traces $\operatorname{tr}[\mathcal{O}(k)]^{\Gamma_{1} \Gamma_{2} ; \beta \beta^{\prime}}=\sum_{\alpha}\left\langle\Gamma_{1} \beta \Gamma_{2} \alpha|\mathcal{O}(k)| \Gamma_{1} \beta^{\prime} \Gamma_{2} \alpha\right\rangle$. However, quite often the trace of this trace matrix or its average, as given by Eq. (1), which is important in applications, may not propagate in a simple manner. There are approximate methods for propagating trace of the trace matrix and they are significant in particular when the integrity basis contains far too many operators [18]. A very important example here is fixed- $L$ averages in IBM's or fixed- $J$ (and $J T$ ) averages in the shell model. For these, it is not possible to write a simple propagation equation in terms of the defining space averages. On the other hand traces over irreps of group symmetries (higher than $J$ symmetry) can be propagated in many situations using Casimir invariants. French and Draayer [19] showed that by simple counting of irreps of $G_{2}$ in $G_{1}$ and the scalars one can construct in terms of the Casimir invariants of $G_{1}$ and $G_{2}$ will immediately confirm if propagation via Casimir invariants is possible; in this situation the integrity 
basis reduces to Casimir operators of $G_{1}$ and $G_{2}$. In this paper we restrict ourselves to examples in IBM's where this result applies; Refs. [20,21] give first IBM examples.

For IBM's the SGA, called $G_{1}$ above, is $U(\mathcal{N})$, with $\mathcal{N}=6$ for sdIBM, 15 for sdgIBM etc. and its irreps $\Gamma_{1}$ are labeled uniquely by the boson number $m$ as all $m$ boson states are symmetric with respect to $U(\mathcal{N})$. Now, consider the average of an operator $\mathcal{O}(k)$ over the irreps $\left(m, \Gamma_{2}\right)$ with $\Gamma_{2}$ 's being the irreps of a subalgebra $G_{2}$ of $U(\mathcal{N})$. Say the the number of $\left(m, \Gamma_{2}\right)$ 's, called $\Gamma^{i}$ 's hereafter, for $m \leq k$ is $r$. Also assume that there are $r$ number of invariants $\hat{\mathcal{C}}_{i}, i=1,2, \ldots, r$ of maximum body rank $k$ constructed out of the products of $m$ and the Casimir invariants of $G_{2}$. Then, for any irrep $\Gamma^{0}$, clearly $\langle\mathcal{O}\rangle^{\Gamma^{0}}=\sum_{i=1}^{r} a_{i}\left\langle\hat{\mathcal{C}}_{i}\right\rangle^{\Gamma^{0}}$ where $a_{i}$ are constants. The $a_{i}$ 's can be determined by assuming that the averages $\langle\mathcal{O}\rangle^{\Gamma^{j}}$ are known for the irreps $\Gamma^{j}$, $j=1,2, \ldots, r$. For example, $\Gamma_{j}$ 's can be chosen to be the irreps $\left(m, \Gamma_{2}\right)$ 's for $m \leq k$. With this, defining the row matrices $[\mathcal{C}]$ and $\left[\mathcal{O}_{i n p}\right]$ and the $r \times r$ matrix $[X]$ as

$$
[\mathcal{C}] \Leftrightarrow \mathcal{C}_{i}=\left\langle\hat{\mathcal{C}}_{i}\right\rangle^{\Gamma^{0}}, \quad\left[\mathcal{O}_{\text {inp }}\right] \Leftrightarrow \mathcal{O}_{\text {inp:i }}=\langle\mathcal{O}\rangle^{\Gamma^{i}}, \quad[X] \Leftrightarrow X_{i j}=\left\langle\hat{\mathcal{C}}_{j}\right\rangle^{\Gamma^{i}}
$$

the propagation equation is

$$
\langle\mathcal{O}\rangle^{\Gamma}=[\mathcal{C}][X]^{-1}\left[\widetilde{\mathcal{O}_{i n p}}\right]
$$

As the eigenvalues of the Casimir invariants of the algebras $U(\mathcal{N}), O(\mathcal{N})$ etc. are known, construction of $[\mathcal{C}]$ and $[X]$ is easy. In the reminder of this paper the $H$ is assumed to be $(1+2)$-body. As an example let us consider $S U(3)$ centroids in $s d$ IBM. Here $G_{1}=U(6)$ and $G_{2}=S U(3)$. Simple counting of scalar in terms of the number operator $\hat{n}$ and the quadratic Casimir operator $\hat{\mathcal{C}}_{2}$ and the cubic Casimir operator $\hat{\mathcal{C}}_{3}$ of $\mathrm{SU}(3)$, confirm that they exhaust all the scalars needed for propagating $\langle\mathcal{O}(k)\rangle^{m,(\lambda \mu)}$ for any $k[18,21]$. Note that $(\lambda \mu)^{\prime}$ 's denote $S U(3)$ irreps. Propagation equation for the energy centroids over $S U(3)$ irreps can be written as $\langle H\rangle^{m,(\lambda \mu)}=$ $a_{0}+a_{1} m+a_{2} m^{2}+a_{3} \mathcal{C}_{2}(\lambda \mu)$ where 


$$
\mathcal{C}_{2}(\lambda \mu)=\left\langle(\lambda \mu) \alpha\left|\hat{\mathcal{C}}_{2}\right|(\lambda \mu) \alpha\right\rangle=\left[\lambda^{2}+\mu^{2}+\lambda \mu+3(\lambda+\mu)\right] .
$$

Using Eqs. (3) and (4), the propagation equation, in terms of the energy centroids for $m \leq 2$, is [21]

$$
\begin{aligned}
\langle H\rangle^{m,(\lambda \mu)}= & \frac{1}{2}\left(2-3 m+m^{2}\right)\langle H\rangle^{0,(00)}+\left(2 m-m^{2}\right)\langle H\rangle^{1,(20)} \\
& +\left[-\frac{5}{6} m+\frac{5}{18} m^{2}+\frac{1}{18} \mathcal{C}_{2}(\lambda \mu)\right]\langle H\rangle^{2,(40)} \\
& +\left[\frac{1}{3} m+\frac{2}{9} m^{2}-\frac{1}{18} \mathcal{C}_{2}(\lambda \mu)\right]\langle H\rangle^{2,(02)}
\end{aligned}
$$

Eq. (5) extends easily to the $S U(3)$ limit of $p f$ IBM with $U(10)$ SGA but not to $s d g$, sdgpf, etc. IBM's. Now we will derive 3 new propagation equations for energy centroids.

In the $U(\mathcal{N}) \supset \sum_{i}\left[U\left(\mathcal{N}_{i}\right) \supset O\left(\mathcal{N}_{i}\right)\right] \oplus$ symmetry limits of IBM's, with the bosons carrying angular momenta $\ell_{1}, \ell_{2}, \ldots$ so that $\mathcal{N}_{i}=\left(2 \ell_{i}+1\right)$ and $\mathcal{N}=\sum_{i} \mathcal{N}_{i}$, for a given $i^{\text {th }}$ orbit, $U\left(\mathcal{N}_{i}\right)$ generates number of particles $m_{i}$ in the orbit and $O\left(\mathcal{N}_{i}\right)$ generates the corresponding seniority quantum number $\omega_{i}$. The number operators $\hat{n}_{i}$ of $U\left(\mathcal{N}_{i}\right)$ and the quadratic Casimir operators of $O\left(\mathcal{N}_{i}\right)$ or the corresponding pairing operators $\hat{P}_{2}\left(O\left(\mathcal{N}_{i}\right)\right)$ suffice to give fixed $\widetilde{m} \widetilde{\omega}=\left(m_{1} \omega_{1}, m_{2} \omega_{2}, \ldots\right)$ averages of $H$. Appendix A in Ref. [22] gives the explicit form of $\hat{P}_{2}\left(O\left(\mathcal{N}_{i}\right)\right)$ for a general situation. Fixed$\widetilde{m} \widetilde{\omega}$ centroids of $H$ can be written as $\langle H\rangle^{\tilde{m} \widetilde{\omega}}=\sum_{i} m_{i} \epsilon_{i}+\sum_{i \geq j} a_{i j} m_{i}\left(m_{j}-\delta_{i j}\right)+$ $\sum_{i} c_{i}\left\langle\hat{P}_{2}\left(O\left(\mathcal{N}_{i}\right)\right)\right\rangle^{m_{i} \omega_{i}}$. Solving for $a_{i j}$ 's and $c_{i}$ 's in terms of the centroids for $m \leq 2$, the final propagation equation, for IBM's with no internal degrees of freedom, is

$$
\begin{aligned}
& \langle H\rangle^{\tilde{m} \widetilde{\omega}}=\sum_{i} m_{i} \epsilon_{i}+\sum_{i>j} \overline{V_{i j}} m_{i} m_{j}+\sum_{i} \frac{m_{i}\left(m_{i}-1\right)}{2}\langle V\rangle^{m_{i}=2, \omega_{i}=2} \\
& +\sum_{i} \frac{\langle V\rangle^{m_{i}=2, \omega_{i}=0}-\langle V\rangle^{m_{i}=2, \omega_{i}=2}}{2 \mathcal{N}_{i}}\left(m_{i}-\omega_{i}\right)\left(m_{i}+\omega_{i}+\mathcal{N}_{i}-2\right) ; \\
& \overline{V_{i j}}=\left\{\left[\mathcal{N}_{i}\left(\mathcal{N}_{j}+\delta_{i j}\right)\right] /\left(1+\delta_{i j}\right)\right\}^{-1} \sum_{L} V_{\ell_{i} \ell_{j} \ell_{i} \ell_{j}}^{L}(2 L+1), \\
& \langle V\rangle^{m_{i}=2, \omega_{i}=0}=\left\langle\left(\ell_{i} \ell_{i}\right) L_{i}=0|V|\left(\ell_{i} \ell_{i}\right) L_{i}=0\right\rangle, \\
& \langle V\rangle^{m_{i}=2, \omega_{i}=2}=\left[\frac{\mathcal{N}_{i}\left(\mathcal{N}_{i}+1\right)}{2} \overline{V_{i i}}-\langle V\rangle^{m_{i}=2, \omega_{i}=0}\right] /\left[\frac{\mathcal{N}_{i}\left(\mathcal{N}_{i}+1\right)}{2}-1\right] .
\end{aligned}
$$


Note that in Eqs. (6), $\epsilon_{i}$ are energies of the single particle levels with angular momentum $\ell_{i}$ and $V_{\ell_{i} \ell_{j} \ell_{i} \ell_{j}}^{L}=\left\langle\left(\ell_{i} \ell_{j}\right) L|V|\left(\ell_{i} \ell_{j}\right) L\right\rangle$ are two particle matrix elements of the two-body part of $H$. Also in Eq. (6), for $s$ orbit $m_{s}=2$ and $\omega_{s}=2$ and there will be no two-boson state with $\omega_{s}=0$. Eq. (6) for $s d g \mathrm{IBM}$ is given first in [23], i.e. for averages over the irreps of the algebras in the chain $U_{s d g}(15) \supset U_{s}(1) \oplus\left[U_{d}(5) \supset O_{d}(5)\right] \oplus\left[U_{g}(9) \supset O_{g}(9)\right]$. Similarly Eq. (6) gives $H$ averages over the irreps of $U_{s d}(6) \supset U_{d}(5) \supset O_{d}(5)$ of $s d \mathrm{IBM}, U_{s d p f}(16) \supset\left[U_{d}(5) \supset\right.$ $\left.O_{d}(5)\right] \oplus\left[U_{p}(3) \supset O_{p}(3)\right] \oplus\left[U_{f}(7) \supset O_{f}(7)\right]$ of $s d p f$ IBM etc. Moreover this extends easily (this will be discussed elsewhere) to IBM's with internal degrees of freedom. Let us add that it is also possible to write down propagation equations for the variances $\left\langle\left[H-\langle H\rangle^{\tilde{m} \tilde{\omega}}\right]^{2}\right\rangle^{\tilde{m} \widetilde{\omega}}$ using the results in $[20,24]$.

In IBM- $T$ with $U(3 \mathcal{N}) \supset U(\mathcal{N}) \otimes\left[S U_{T}(3) \supset O_{T}(3)\right]$ where $U(\mathcal{N})$ gives the spatial part (for $s d, s d g, s d p f$ etc.) and $O_{T}(3)$ generating isospin [25], it is possible to propagate the centroids $\langle H\rangle^{m,\{f\}, T} \equiv\langle H\rangle^{m,(\lambda \mu), T}$. Note that the $U(\mathcal{N})$ irreps are labeled by $\{f\}=\left\{f_{1}, f_{2}, f_{3}\right\}$ where $f_{1} \geq f_{2} \geq f_{3} \geq 0$ and $m=f_{1}+f_{2}+f_{3}$. The corresponding $S U_{T}(3)$ irreps are $(\lambda, \mu)=\left(f_{1}-f_{2}, f_{2}-f_{3}\right)$. The $S U_{T}(3)$ to $O_{T}(3)$ reductions follow from the formulas given by Elliott [26,27]. The scalars $1, \hat{n}, \hat{n}^{2}$, $\hat{\mathcal{C}}_{2}\left(S U_{T}(3)\right)$ and $\hat{T}^{2}$ and the energy centroids for $m \leq 2$, via Eqs. (2), (3) and (4), give

$$
\begin{aligned}
\langle H\rangle^{m,(\lambda \mu), T}= & {\left[1-\frac{3}{2} m+\frac{m^{2}}{2}\right]\langle H\rangle^{0,(00), 0}+\left[2 m-m^{2}\right]\langle H\rangle^{1,(10), 1} } \\
& +\left[-\frac{1}{6} m+\frac{1}{18} m^{2}+\frac{1}{9} \mathcal{C}_{2}(\lambda \mu)-\frac{1}{6} T(T+1)\right]\langle H\rangle^{2,(20), 0} \\
& +\left[-\frac{5}{6} m+\frac{5}{18} m^{2}+\frac{1}{18} \mathcal{C}_{2}(\lambda \mu)+\frac{1}{6} T(T+1)\right]\langle H\rangle^{2,(20), 2} \\
& +\left[\frac{1}{2} m+\frac{1}{6} m^{2}-\frac{1}{6} \mathcal{C}_{2}(\lambda \mu)\right]\langle H\rangle^{2,(01), 1} .
\end{aligned}
$$

For $s d$ IBM- $T$, starting with the general hamiltonian given in Appendix-A of [27] which contains the $s$ and $d$ boson energies and 17 two-particle matrix elements $V_{\ell_{l} \ell_{2} \ell_{3} \ell_{4}}^{L, t}$, it is easy to write down $\langle H\rangle^{m,(\lambda \mu), T}$ for $m \leq 2$; for $m=2$ the two-boson isospins $t$ uniquely 
define the corresponding $S U_{T}(3)$ irreps. Thus Eq. (7) for $\langle H\rangle^{m,(\lambda \mu), T}$ is easy to apply for any $m$.

In IBM- $S T$, a group chain of interest is $[28] U(6 \mathcal{N}) \supset U(\mathcal{N}) \otimes\left[S U_{S T}(6) \supset O_{S T}(6)\right]$ with $U(\mathcal{N})$ generating the spatial part and $S U_{S T}(6)$ or $U_{S T}(6)$ ] generating spinisospin part; note that the Wigner's spin-isospin super-multiplet algebra $S U_{S T}(4)$ is isomorphic to $O_{S T}(6)$. Just as before, it is possible to propagate the centroids $\langle H\rangle^{m,\{f\},[\sigma]}$. Here $\{f\}^{\prime}$ 's are the irreps of $U(\mathcal{N})$ or equivalently $U_{S T}(6)$ and $\{f\}=$ $\left\{f_{1}, f_{2}, f_{3}, f_{4}, f_{5}, f_{6}\right\}$ where $\sum_{i} f_{i}=m$ and $f_{i} \geq f_{i+1} \geq 0$. The $O_{S T}(6)$ irreps are labeled by $[\sigma]=\left[\sigma_{1}, \sigma_{2}, \sigma_{3}\right]$ and the $\{f\}$ to $[\sigma]$ reductions, needed for the results discussed ahead, follow from the analytical formulas given in [27] and the tabulations in [29]. Eqs. (2) and (3) give, using the quadratic Casimir invariants $\hat{\mathcal{C}}_{2}$ 's of $U_{S T}(6)$ and $O_{S T}(6)$,

$$
\begin{aligned}
\langle H\rangle^{m,\{f\},[\sigma]}= & {\left[1-\frac{3}{2} m+\frac{m^{2}}{2}\right]\langle H\rangle^{0,\{0\},[0]}+\left[2 m-m^{2}\right]\langle H\rangle^{1,\{1\},[1]} } \\
& +\left[-\frac{5}{3} m+\frac{1}{4} m^{2}+\frac{1}{6} \mathcal{C}_{2}(\{f\})+\frac{1}{12} \mathcal{C}_{2}([\sigma])\right]\langle H\rangle^{2,\{2\},[2]} \\
& +\left[-\frac{1}{12} m+\frac{1}{12} \mathcal{C}_{2}(\{f\})-\frac{1}{12} \mathcal{C}_{2}([\sigma])\right]\langle H\rangle^{2,\{2\},[0]} \\
& +\left[\frac{5}{4} m+\frac{1}{4} m^{2}-\frac{1}{4} \mathcal{C}_{2}(\{f\})\right]\langle H\rangle^{2,\left\{1^{2}\right\},\left[1^{2}\right]}
\end{aligned}
$$

where $\mathcal{C}_{2}(\{f\})=\left\langle\hat{\mathcal{C}}_{2}\left(U_{S T}(6)\right)\right\rangle^{\{f\}}=\sum_{i=1}^{6} f_{i}\left(f_{i}+7-2 i\right)$ and $\mathcal{C}_{2}([\sigma])=$ $\left\langle\hat{\mathcal{C}}_{2}\left(O_{S T}(6)\right)\right\rangle^{[\sigma]}=\sum_{i=1}^{3} \sigma_{i}\left(\sigma_{i}+6-2 i\right)$. Diagonalizing $\hat{\mathcal{C}}_{2}\left(O_{S T}(6)\right)$ in the $\left|\left(\ell_{1} \ell_{2}\right) L S T\right\rangle$ basis and applying the resulting unitary transformation to the $H$ matrix in this basis will give the input averages in Eq. (8).

Now we will apply Eqs. (5)-(8) to study regularities generated by random interactions in energy centroids. In all the calculations used are independent Gaussian random variables with zero center and unit variance and a 1000 member ensemble. We begin with the simplest example of $s d \mathrm{IBM}$ centroids. The highest $S U(3)$ irrep for a given $m$ is $(2 m, 0)$ and Eq. (5) gives, $\langle H\rangle^{m,(\lambda \mu)}-\langle H\rangle^{m,(2 m, 0)}=\left[\mathcal{C}_{2}(\lambda \mu)-\mathcal{C}_{2}(2 m, 0)\right] \Delta / 18$ 
with $\Delta=\langle H\rangle^{2,(40)}-\langle H\rangle^{2,(02)}$. Therefore the probability of finding $\Delta$ to be positive or negative will simply give the probability for finding the highest or lowest $m$ particle $S U(3)$ irrep to be lowest in energy. With the two-particle matrix elements chosen to be Gaussian variables (with zero center and unit variance), $\Delta$ itself will be a Gaussian variable with zero center. For $m=3 k, 3 k+1$ and $3 k+2, k$ being a positive integer, the lowest $S U(3)$ irreps are (00), (20) and (02) respectively. They will be lowest in energy with $50 \%$ and the $(2 m, 0)$ irrep will be lowest in energy with $50 \%$ probability. Thus, it is easy to understand the regularities in centroids defined over fixed $S U(3)$ irreps in $s d$ IBM with one plus two-body hamiltonians, without constructing the many boson hamiltonian matrix but just by using the propagation equation (5).

In $s d g$ IBM, regularities in fixed- $\left(m_{s}, m_{d}, v_{d}, m_{g}, v_{g}\right)$ centroids are studied using the propagation Eq. (6). Choosing the 3 single particle energies $\left(\epsilon_{s}, \epsilon_{d}, \epsilon_{g}\right)$ and the 16 diagonal two-particle matrix elements $V_{\ell_{1} \ell_{2} \ell_{1} \ell_{2}}^{L}$, with $\ell_{i}=0,2$ and 4 to be Gaussian variables, the probability for the centroid of a given $\left(m_{s}, m_{d}, v_{d}, m_{g}, v_{g}\right)$ configuration to be lowest is calculated for $m=6-25$ and the results are shown in Fig. 1 for $m=15$. To maintain proper scaling, the $\epsilon^{\prime}$ 's are divided by $m$ and the $V^{L}$ by $m(m-1)$ just as in [2]. For the discussion of the results we define $\pi(x)$ such that $\pi(x)=0$ for $x$ even and $\pi(x)=1$ for $x$ odd. It is seen from Fig. 1 , and also valid for any $m$, that the configurations $\left(m_{s}, m_{d}=v_{d}=m-m_{s}, m_{g}=v_{g}=0\right)$, $\left(m_{s}, m_{d}=m-m_{s}, v_{d}=\pi\left(m_{d}\right), m_{g}=v_{g}=0\right),\left(m_{s}, m_{d}=v_{d}=0, m_{g}=v_{g}=m-m_{s}\right)$ and $\left(m_{s}, m_{d}=v_{d}=0, m_{g}=m-m_{s}, v_{g}=\pi\left(m_{g}\right)\right)$ exhaust about $91 \%$ probability. Moreover, the configurations with $m_{s}=m_{d}=0$ carry $\sim 20 \%, m_{s}=m_{g}=0$ carry $\sim 21 \%, m_{s}=m$ carries $\sim 24 \%$ and $m_{s} \neq 0$ but $m_{d}=0$ or $m_{g}=0$ carry $\sim 26 \%$ probability. Thus the $m_{s}=m$ configuration and the four configurations with $m_{s}=0$ are most probable to be lowest in energy. However, other configurations with $m_{s} \neq$ 
$0, m$ (they are 49 out of 1195 , configurations in the $m=15$ example) give nonnegligible probability for being lowest. Thus about $\sim 4 \%$ of the $\left(m_{s}, m_{d}, v_{d}, m_{g}, v_{g}\right)$ configurations will have probability to be lowest with random interactions.

For $s d$ IBM-T, it is easily seen from Eq. (7) that the one-body part of $H$ will not play any role in the study of fixed- $(\lambda, \mu) T$ centroids. Choosing $V_{\ell_{1} \ell_{2} \ell_{1} \ell_{2}}^{L, t}$ 's to be Gaussian variables, the centroids are generated, using Eq. (7), for $m=10-25$ and for all allowed $(\lambda, \mu) T$. Some typical results for the regularities are shown in Fig. 2. Firstly, for a given $m$ the highest $S U_{T}(3)$ irrep is $(m, 0)$ with $T_{\max }=m$ and $T_{\text {min }}=\pi(m)$. For $m=3 k, 3 k+1$ and $3 k+2$, with $k$ being a positive integer, the lowest $S U_{T}(3)$ irreps are (00), (10) and (01) with $T=0,1$ and 1 respectively; for the later two situations the next lowest irreps are (02) and (20) respectively with $T_{\min }=0$. For $m=3 k$, it is seen from Fig. 2 that the lowest $S U_{T}(3)$ irrep's centroid (here $T$ is unique) is lowest with $\sim 35 \%$ probability. Similarly the highest irreps centroid is lowest with $\sim 60 \%$ probability and this splits into $\sim 30 \%$ each for the lowest and highest $T$ 's. For $m=3 k+1$ and $3 k+2$, the probability for the centroid of the highest irrep to be lowest in energy is same as for $m=3 k$. However for the centroid of the lowest irrep, the probability is $\sim 29 \%$ and the next lowest irrep appears with $\sim 6 \%$. Thus in general the centroids of the highest and the lowest (for $m=3 k+1$ and $3 k+2$, the lowest two) $S U_{T}(3)$ irreps exhaust about $95 \%$ of the probability for being lowest in energy. As the two particle centroids $X^{t}=\langle H\rangle^{m=2, t}$ are linear combinations of $V^{\prime}$ s, it can be seen that they themselves are Gaussian variables. Note that $\langle H\rangle^{m,(\lambda \mu) T}-\langle H\rangle^{m,(m, 0) m}=\left[\mathcal{C}_{2}(\lambda \mu)-\mathcal{C}_{2}(m, 0)\right] \Delta_{1}+[T(T+1)-m(m+1)] \Delta_{2}$ where $\Delta_{1}=\frac{1}{9} X^{0}+\frac{1}{18} X^{2}-\frac{1}{6} X^{1}$ and $\Delta_{2}=\frac{1}{6}\left(X^{2}-X^{0}\right)$. Calculations with $X^{t}$ 's taken as Gaussian variables with same variance (actually the variance of $X^{0}$ and $X^{2}$ are same and that of $X^{1}$ is $\sim 20 \%$ higher) are carried out and it is seen that they give almost same results as in Fig. 2. 
For $s d \mathrm{IBM}-S T$, as seen from Eq. (8), the energy centroids $\langle H\rangle^{m,\{f\},[\sigma]}$ are determined by the 2-particle averages $\langle H\rangle^{2,\{2\},[2]},\langle H\rangle^{2,\{2\},[0]}$ and $\langle H\rangle^{2,\left\{1^{2}\right\},\left[1^{2}\right]}$ and they are linear combinations of the two particle matrix elements $V^{L S T}$ in the $\left|\left(\ell_{1} \ell_{2}\right) L S T\right\rangle$ basis. Instead of choosing $V^{L S T}$ to be Gaussian variables, we have chosen, using the result found in the $s d \mathrm{IBM}-T$ examples, the three 2-particle averages to be Gaussian variables. Using this, the probabilities are calculated for various $m$ values and some of the results are shown in Fig. 3. Firstly for a given $m$ the highest $\{f\}$ is $\{m\}$. The corresponding highest and lowest $[\sigma]$ are $[m]$ and $[\pi(m)]$. For all $m$ the centroid of the highest $U_{S T}(6)$ irrep is lowest with $\sim 56 \%$ probability and this splits into $\sim 34 \%$ and $\sim 22 \%$ for the highest and lowest $\left.O_{S T} 6\right)$ irreps. For $m=6 k, 6 k \pm 1,6 k \pm 2$ and $6 k+3$, with $k$ a positive integer, the lowest $U_{S T}(6)$ irreps are those that can be reduced to the irreps $\{0\},\{1\},\left(\{2\},\left\{1^{2}\right\}\right)$ and $\left(\left\{1^{3}\right\},\{21\}\right)$ respectively. These irreps with the corresponding lowest $[\sigma]$ are lowest with probability $\sim 43 \%$.

In conclusion, with random interactions, the lowest and highest group irreps (i.e. irreps of $G_{2}$ in $G_{1} \supset G_{2}$ ) carry most of the probability for the corresponding centroids to be lowest in energy. With the inclusion of a subalgebra $\left(G_{1} \supset G_{2} \supset G_{3}\right)$, these probabilities split into the probabilities for the corresponding lowest and highest irreps of the subalgebra. This is indeed the situation for all the examples discussed in this paper. Continuing with the process of embedding subalgebras, the $O(3)$ algebra generating $L$ can be reached (with generalization for systems with $L T, L S T$ or $J T$ ). Then clearly the energy centroids of highest and lowest $L$ 's should be most probable and this is found to be true numerically in $[11,12]$. An important aspect of the energy centroids is that they propagate via Casimir invariants in many situations. New propagation equations are derived in this paper (Eqs. (6), (7) and (8)). In fact there are many other situations where such equations can be derived; an example is for the centroids over the irreps $\left[m_{s d}\left(\lambda_{s d} \mu_{s d}\right) ; m_{p f}\left(\lambda_{p f} \mu_{p f}\right)\right]$ of $\left[U_{s d}(6) \supset S U_{s d}(3)\right] \oplus$ 
$\left[U_{p f}(10) \supset S U_{p f}(3)\right]$ algebra of $s d p f$ IBM [30]. These will be discussed in a longer paper along with extensions of the present work to spectral variances and also to shell model symmetries. Finally, an important observation is that the propagators carry information about $G_{1} \supset G_{2}$ geometry (i.e. $G_{1} \supset G_{2}$ reduced Wigner coefficients and $G_{2}$ Racah coefficients) and thus it is plausible that propagation equations may be useful in quantifying geometric chaos. This is being investigated and it should be remarked that only recently the role of Wigner-Racah algebra in two-body random matrix ensembles is established [31].

Thanks are due to Y.M. Zhao for useful correspondence and for making available Ref. [12] before it is submitted for publication. 


\section{REFERENCES}

[1] C.W. Johnson, G.F. Bertsch, and D.J. Dean, Phys. Rev. Lett. 80, 2749 (1998).

[2] R. Bijker and A. Frank, Phys. Rev. Lett. 84, 420 (2000).

[3] V. Velázquez, J.G. Hirsch, A. Frank, and A.P. Zuker, Phys. Rev. C 67, 034311 (2003); Y.M. Zhao, A. Arima, N. Shimizu, K. Ogawa, N. Yoshinaga, and O. Scholten, ibid. 70, 054322 (2004).

[4] D. Mulhall, A. Volya, and V. Zelevinsky, Phys. Rev. Lett. 85, 4016 (2000).

[5] V.K.B. Kota and K. Kar, Phys. Rev. E 65, 026130 (2002).

[6] V. Zelevinsky and A. Volya, Phys. Rep. 391, 311 (2004).

[7] R. Bijker and A. Frank, Phys. Rev. C 64, 061303 (2001).

[8] Y.M. Zhao, A. Arima, and N. Yoshinaga, Phys. Rev C 66, 034302 (2002).

[9] V.K.B. Kota, High Energy Phys. \& Nucl. Phys. (China), 28, 1307 (2004); nuclth/0401038.

[10] Y.M. Zhao, A. Arima, and N. Yoshinaga, Phys. Rep. 400, 1 (2004).

[11] Y.M. Zhao, A. Arima, and N. Yoshinaga, Phys. Rev. C 66, 064323 (2002).

[12] Y.M. Zhao, A. Arima, and K. Ogawa, Phys. Rev. C 71, 017304 (2005).

[13] T. Papenbrock and H.A. Weidenmüller, Phys. Rev. Lett. 93, 132503 (2004).

[14] J.B. French, in Isospin in Nuclear Physics, edited by D.H.Wilkinson (North Holland, Amsterdam, 1969), p.259; F.S. Chang, J.B. French, and T.H. Thio, Ann. Phys. (N.Y.) 66, 137 (1971).

[15] J.C. Parikh, Group Symmetries in Nuclear Structure (Plenum, New York, 1978); 
S.S.M. Wong, Nuclear Statistical Spectroscopy (Oxford University Press, New York, 1986).

[16] C. Quesne, J. Math. Phys. 16, 2427 (1975).

[17] J.P. Draayer and G. Rosensteel, Nucl. Phys. A439, 61 (1985).

[18] V.K.B. Kota and K. Kar, Pramana-J. Phys. 32, 647 (1989).

[19] J.B. French and J.P. Draayer, in Group Theoretical Methods in Physics, edited by W. Beiglbock et. al. (Springer, Berlin, 1979), p.394.

[20] V.K.B. Kota, J. de Physique Letters 40, L579 (1979); 41, L143 (1980).

[21] V.K.B. Kota, Ann. Phys. (N.Y.) 134, 221 (1981).

[22] V.K.B. Kota, Ann. Phys. (N.Y.) 280, 1 (2000).

[23] Y.D. Devi and V.K.B. Kota, Physical Research Laboratory (Ahmedabad, India) Technical Report PRL-TN-90-68 (1990).

[24] C. Quesne and S. Spitz, Ann. Phys. (N.Y.) 112, 304 (1978).

[25] J.P. Elliott and A.P. White, Phys. Lett. B97, 169 (1980).

[26] J.P. Elliott, Proc. Roy. Soc. (London) A245, 128 (1958).

[27] V.K.B. Kota, Ann. Phys. (N.Y.) 265, 101 (1998).

[28] J.P. Elliott and J.A. Evans, Phys. Lett. B101, 216 (1981).

[29] B.G. Wybourne, Symmetry Principles and Atomic Spectroscopy (Wiley Interscience, New York, 1970).

[30] J. Engel and F. Iachello, Phys. Rev. Lett. 54, 1126 (1985).

[31] V.K.B. Kota, J. Math. Phys., in press; Z. Pluhar and H.A. Weidenmüller, Ann. 
Phys. (N.Y.) 297, 344 (2002). 


\section{FIGURES}

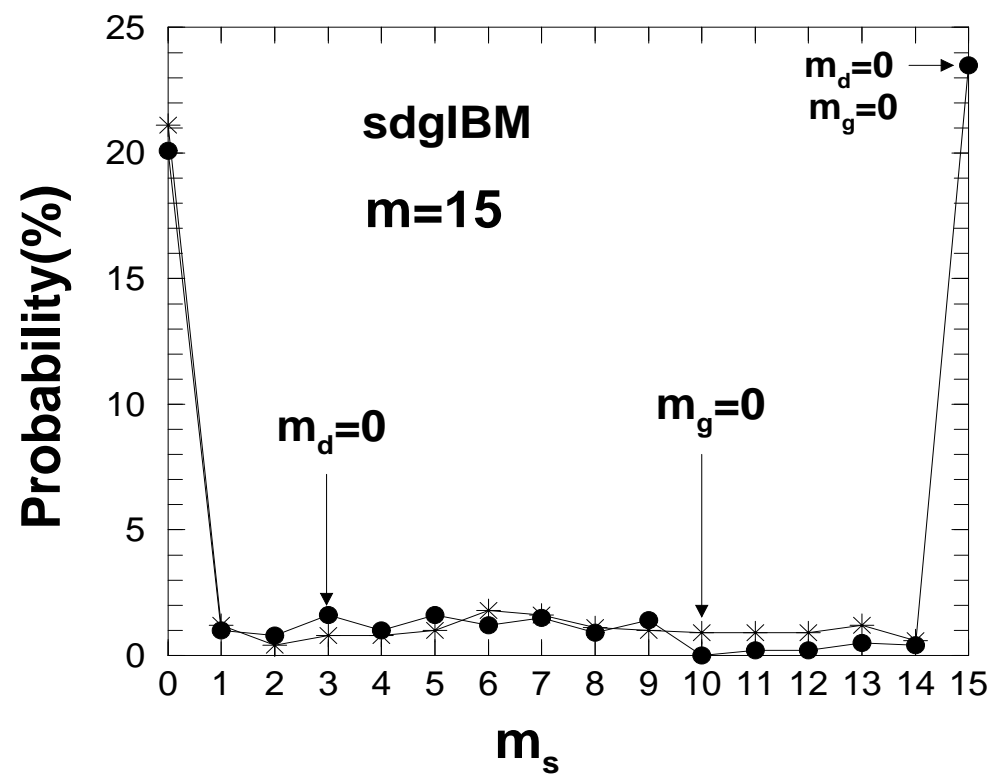

FIG. 1. Probabilities for $s d g$ IBM fixed- $\left(m_{s}, m_{d}, v_{d}, m_{g}, v_{g}\right)$ centroid energies to be lowest in energy vs $m_{s}$ for a system of 15 bosons $(m=15)$. For each $m_{s}$, the probability shown is the sum of the probabilities for the irreps with the seniority quantum number lowest $\left(v_{\ell}=\pi\left(m_{\ell}\right)\right)$ and highest $\left(v_{\ell}=m_{\ell}\right)$. Filled circles and stars are for configurations with $m_{d}=0$ and $m_{g}=0$ respectively; they are joined by lines to guide the eye. Note that for $m_{s}=15$ both $m_{d}=0$ and $m_{g}=0$. 


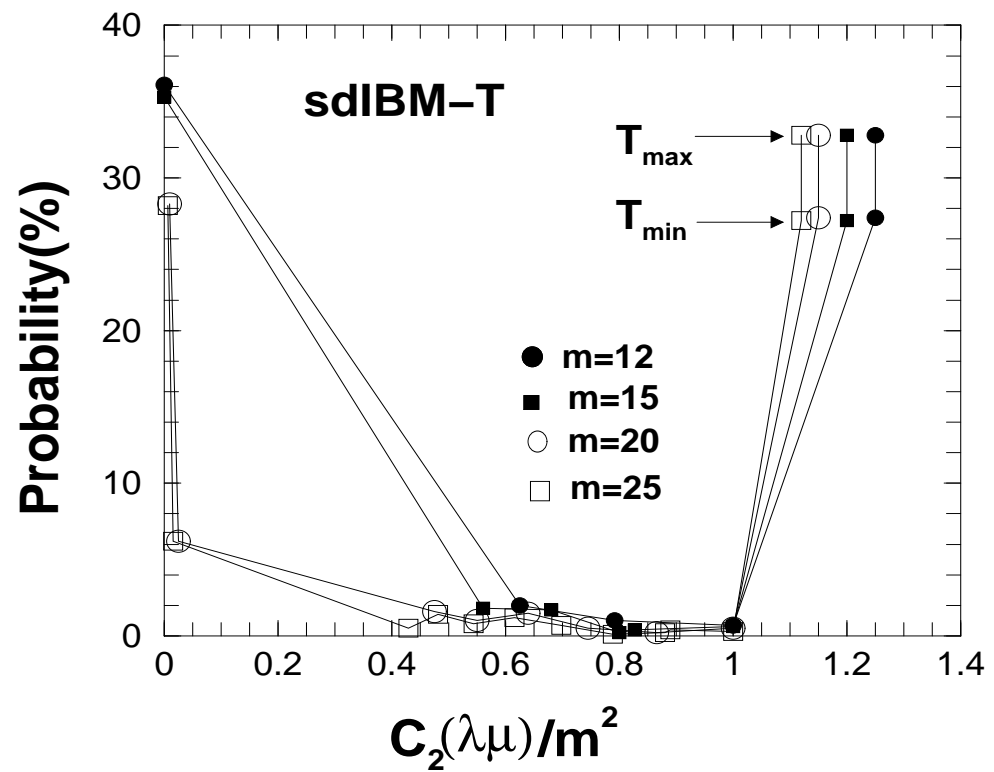

FIG. 2. Probabilities for the $s d$ IBM-T's $(\lambda \mu) T$ centroid energies to be lowest in energy vs $\mathcal{C}_{2}(\lambda \mu) / m^{2}$ for boson systems with $m=12,15,20$ and 25 . Except for the highest $(\lambda \mu)$, for all other $(\lambda \mu)$ 's shown in the figure, the probabilities are for $T_{\max }$ if $\lambda \neq 0$ and $\mu \neq 0$ and they are for $T_{\min }$ if $\lambda=0$ or $\mu=0$. For the irreps not shown in the figure, the probability is $<0.1 \%$. All the points for a given $m$ are joined by lines to guide the eye. 


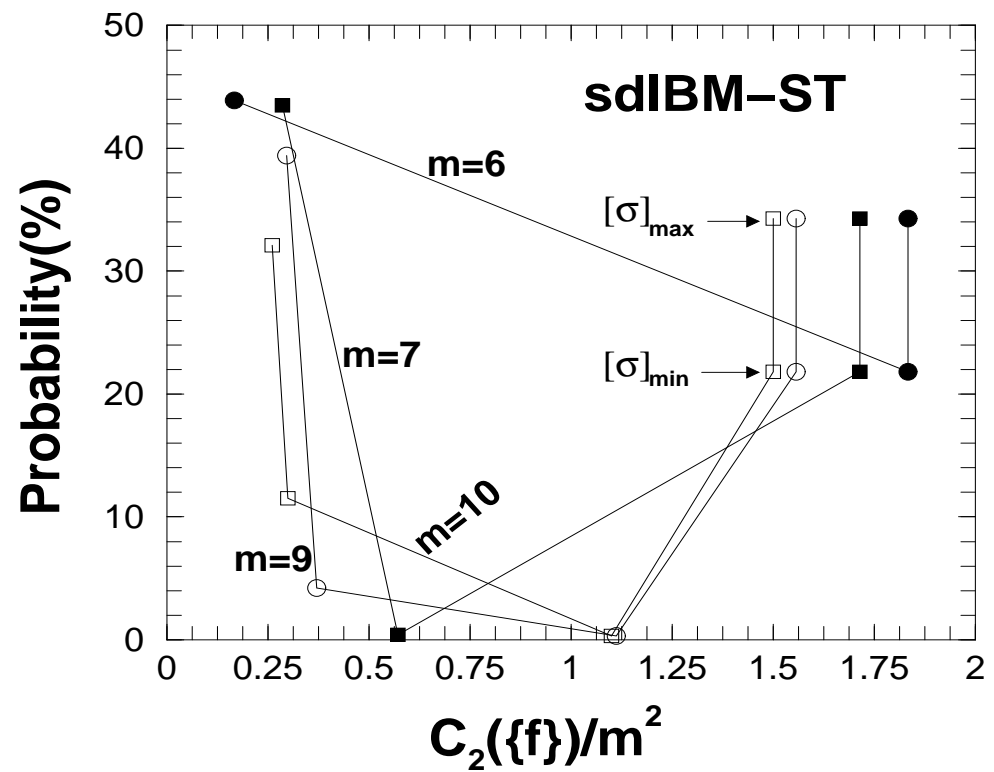

FIG. 3. Probabilities for the $s d$ IBM-ST's $\{f\}[\sigma]$ centroid energies to be lowest in energy vs $\mathcal{C}_{2}(\{f\}) / m^{2}$ for boson systems with $m=6,7,9$ and 10 . For the structure of the irreps with probability $>2 \%$, see text. For $m=7,9$ and 10 there is one additional irrep with $\sim 0.3 \%$ probability. For the irreps not shown in the figure, the probability is $<0.1 \%$. All the points for a given $m$ are joined by lines to guide the eye. 